\title{
Oil components modulate the skin delivery of 5-aminolevulinic acid and its ester prodrug from oil-in-water and water-in-oil nanoemulsions
}

This article was published in the following Dove Press journal:

International Journal of Nanomedicine

4 April 201 I

Number of times this article has been viewed

\author{
Li-Wen Zhang' \\ Saleh A Al-Suwayeh ${ }^{2}$ \\ Chi-Feng Hung ${ }^{3}$ \\ Chih-Chieh Chen' \\ Jia-You Fang 1,2,4
}

'Pharmaceutics Laboratory, Graduate Institute of Natural Products, Chang Gung University, Kweishan, Taoyuan, Taiwan; ${ }^{2}$ Department of Pharmaceutics, College of Pharmacy, King Saud University, Riyadh, Saudi Arabia; ${ }^{3}$ School of Medicine, Fu Jen Catholic University, Taipei County, Taiwan; ${ }^{4}$ Department of Cosmetic Science, Chang Gung Institute of Technology, Kweishan, Taoyuan, Taiwan

Correspondence: jia-You Fang Pharmaceutics Laboratory, Graduate Institute of Natural Products, Chang Gung University, 259 Wen-Hwa Ist Road, Kweishan, Taoyuan 333, Taiwan Tel +886-3-2I I 8800 ext 552I

Fax +886-3-2II 8236

Email fajy@mail.cgu.edu.tw
Abstract: The study evaluated the potential of nanoemulsions for the topical delivery of 5-aminolevulinic acid (ALA) and methyl ALA (mALA). The drugs were incorporated in oil-in-water $(\mathrm{O} / \mathrm{W})$ and water-in-oil $(\mathrm{W} / \mathrm{O})$ formulations obtained by using soybean oil or squalene as the oil phase. The droplet size, zeta potential, and environmental polarity of the nanocarriers were assessed as physicochemical properties. The $\mathrm{O} / \mathrm{W}$ and $\mathrm{W} / \mathrm{O}$ emulsions showed diameters of 216-256 and 18-125 nm, which, respectively, were within the range of submicron- and nano-sized dispersions. In vitro diffusion experiments using Franz-type cells and porcine skin were performed. Nude mice were used, and skin fluorescence derived from protoporphyrin IX was documented by confocal laser scanning microscopy (CLSM). The loading of ALA or mALA into the emulsions resulted in slower release across cellulose membranes. The release rate and skin flux of topical drug application were adjusted by changing the type of nanocarrier, the soybean oil $\mathrm{O} / \mathrm{W}$ systems showing the highest skin permeation. This formulation increased ALA flux via porcine skin to $180 \mathrm{nmol} / \mathrm{cm}^{2} / \mathrm{h}$, which was 2.6-fold that of the aqueous control. The CLSM results showed that soybean oil systems promoted mALA permeation to deeper layers of the skin from $\sim 100 \mu \mathrm{m}$ to $\sim 140 \mu \mathrm{m}$, which would be beneficial for treating subepidermal and subcutaneous lesions. Drug permeation from W/O systems did not surpass that from the aqueous solution. An in vivo dermal irritation test indicated that the emulsions were safe for topical administration of ALA and mALA.

Keywords: nanoemulsions, 5-aminolevulinic acid, methyl 5-aminolevulinic acid, skin permeation, soybean oil, squalene

\section{Introduction}

Non-melanoma skin cancers, which include basal cell carcinoma (BCC) and squamous cell carcinoma (SCC), represent the most common malignant neoplasms in humans. ${ }^{1}$ Topical application of 5-aminolevulinic acid (ALA) as a precursor for the photosensitizer, protoporphyrin (Pp) IX, is used in photodynamic therapy (PDT) for treating BCC and SCC. ${ }^{2}$ ALA generates endogenous porphyrins via the heme cycle. Subsequent irradiation of PDT leads to singlet oxygen production and free radicals, causing cellular damage and tissue necrosis. ${ }^{3}$ ALA-PDT is useful for treating skin cancers, actinic keratoses, psoriasis, and acne. ${ }^{4}$ Approaches were taken to increase the cellular uptake by increasing the diffusion of ALA across plasma membranes by using more-lipophilic ALA prodrugs such as methyl ALA (mALA). ${ }^{5}$ mALA also shows less of an inflammatory response in skin compared with its parent form. ${ }^{6}$ Because ALA is a polar compound, the permeability via the skin is low, making it difficult to reach targets in skin tissues. PDT of neoplastic lesions generally uses high 
ALA loadings of $20 \%(\mathrm{w} / \mathrm{w}) .^{7}$ Between $60 \%$ and $80 \%$ of patients in clinical trials experience treatment-related local phototoxicity. Several attempts were made to increase the skin flux of ALA to reduce the dose, such as iontophoresis, permeation enhancers, and patches. ${ }^{8}$ Many carriers are also used to deliver ALA including liposomes, emulsions, and a nanocolloid lotion. ${ }^{9}$

Liposomes have come into focus as a valuable delivery system for ALA. ${ }^{10}$ However, a great disadvantage in using entrapped ALA is the low incorporation efficiency in liposomes. ${ }^{11}$ Moreover, the costs associated with liposomes and their inherent instability may limit their utility. Lipid nano/submicron emulsions are another choice for drug delivery via the skin. Nanoemulsions are a class of emulsions with a droplet size of 20-200 nm. ${ }^{12}$ They do not spontaneously form, and their properties depend on the thermodynamic conditions and preparation methods. Nano/ submicron emulsions are well accepted for their ability to increase skin permeation, prolong action on the skin, and protect the drug from instability. ${ }^{13,14}$

Oil-in-water $(\mathrm{O} / \mathrm{W})$ and water-in-oil $(\mathrm{W} / \mathrm{O})$ emulsions are used in clinical studies, all with therapeutic success in dermatology. The primary aim of this study was to develop and evaluate $\mathrm{O} / \mathrm{W}$ and $\mathrm{W} / \mathrm{O}$ nanoemulsions to increase the absorption of ALA and mALA. Our secondary aim was to elucidate the influence of oil components on drug delivery by the nanocarriers. Soybean oil and squalene were used as the different oils for comparison. The permeation enhancer, $\alpha$-terpineol, was also loaded in the systems. In this report, we describe the physicochemical characterization of the emulsions and the results of in vitro and in vivo studies on the skin permeation of ALA and its prodrug. The skin irritant response to the emulsions was assessed using in vivo methods, including transepidermal water loss (TEWL) and colorimetry.

\section{Experimental Materials}

ALA, mALA, soybean oil, squalene, Pluronic F68 (PF68), $\alpha$-terpineol, Span 80, and Nile red were purchased from Sigma-Aldrich Chemical (St. Louis, MO, USA). Myverol 18-04K (palmitinic acid monoglyceride) was supplied by Quest (Naarden, the Netherlands). Brij 98 was obtained from Acros Organics (Geel, Belgium). Cellulose membranes (Cellu-Sep $^{\circledR}$ T2, with a molecular weight cutoff of 6000-8000) were provided by Membrane Filtration Products (Seguin, TX, USA).

\section{Preparation of $\mathrm{O} / \mathrm{W}$ emulsions}

Lipid emulsions were prepared using hot, high-pressure homogenization and ultrasonication techniques. The oil and aqueous phases were prepared separately. The oil phase consisted of $12 \%(\mathrm{w} / \mathrm{v})$ soybean oil or squalene and $0.3 \%$ Myverol as the lipophilic emulsifier, while the aqueous phase consisted of double-distilled water (DDW) and a hydrophilic emulsifier (2.5\% PF68). ALA or mALA (38 mM) used as the incorporated drug was positioned in the aqueous phase if necessary. Both phases were separately heated to $55^{\circ} \mathrm{C}$ for $15 \mathrm{~min}$. The aqueous phase was added to the oil phase and mixed for $5 \mathrm{~min}$ at $55^{\circ} \mathrm{C}$. The mixture was homogenized in a high-shear homogenizer (Pro 250, Pro Scientific, Monroe, CT, USA) at 12,000 rpm for 10 minutes. The mixture was further treated using a probe-type sonicator (VCX600, Sonics and Materials, Newtown, CT, USA) for 10 minutes at $35 \mathrm{~W}$. The total volume of the resulting product was $10 \mathrm{~mL}$.

\section{Preparation of W/O emulsions}

The oil phase consisted of soybean oil or squalene (44\%) and Span 80 (30\%), while the aqueous phase consisted of water (10\%), Brij 98 (16\%), and the drug. The two phases were separately heated to $55^{\circ} \mathrm{C}$. The oil phase was further mixed using a high-shear homogenizer (Pro 250) for 20 minutes. Then the oil phase was added to the aqueous phase and sonicated using a probe-type sonicator (VCX600) for 10 minutes at $35 \mathrm{~W}$. The total volume of the final product was $10 \mathrm{~mL}$.

\section{Determination of the size and zeta potential}

The mean droplet size (z-average) and zeta potential of the nanoemulsions were measured by photon correlation spectroscopy (Malvern Nano ZS ${ }^{\circledR}$ 90, Malvern Instruments, Worcestershire, UK) using a helium-neon laser with a wavelength of $633 \mathrm{~nm}$. Photon correlations of spectroscopic measurements were carried out at a scattering angle of $90^{\circ}$. The $\mathrm{O} / \mathrm{W}$ emulsions were diluted 1:100 with water before the measurement. In the case of $\mathrm{W} / \mathrm{O}$ emulsions, the formulations were diluted 5-fold with soybean oil or squalene for detection.

\section{Encapsulation efficiency of drugs in the emulsions}

The encapsulation efficiency of ALA and mALA entrapped in the $\mathrm{O} / \mathrm{W}$ emulsions was determined by an ultracentrifugation method. The product was centrifuged at $48,000 \times g$ and $4^{\circ} \mathrm{C}$ 
for 30 minutes in a Beckman Optima $\mathrm{MAX}^{\circledR}$ ultracentrifuge (Beckman Coulter, Fullerton, CA, USA) in order to separate the incorporated drug from the free form. The supernatant and precipitate were analyzed by high-performance liquid chromatography (HPLC) to determine the encapsulation percentage (\%) of the total drug load. The HPLC method for ALA and mALA was described previously. ${ }^{15}$

\section{Molecular environment of the emulsions}

The lipophilic fluorescent marker, Nile red, was used as the model solute, and the molecular environment (polarity) was determined by fluorometric spectrophotometry based on the solvatochromism of Nile red. O/W emulsions with 1 ppm Nile red were prepared as described above. Emission fluorescence spectra were determined with a Hitachi F-2500 spectrometer (Tokyo, Japan). The spectra of emulsions with Nile red were recorded with both slit widths set to $10 \mathrm{~nm}$. The $\lambda_{\text {ex }}$ was fixed at $546 \mathrm{~nm}$, and the emission spectra were recorded from 570 to $700 \mathrm{~nm}$ at a scanning speed of $300 \mathrm{~nm} / \mathrm{min}$.

\section{Animals}

The animal experimental protocol was reviewed and approved by the Institutional Animal Care and Use Committee of Chang Gung University. The committee confirmed that the animal experiment followed the guidelines as set forth by the Guide for Laboratory Factlines and Care. Female nude mice (ICR-Foxn1 nu strain) aged 8 weeks were obtained from the National Laboratory Animal Center (Taipei, Taiwan). Pathogen-free pigs ( 1 week old) were supplied by the Animal Technology Institute Taiwan (Miaoli, Taiwan).

\section{In vitro skin permeation}

The skin permeation of ALA and mALA was measured using a Franz cell assembly. Full-thickness dorsal skin of a pig was mounted between the donor and receptor compartments. A cellulose membrane instead of porcine skin was also used as a barrier to examine drug permeation. The donor consisted of $0.5 \mathrm{~mL}$ of vehicle containing drugs. The receptor medium (5.5 mL) was $\mathrm{pH} 7.4$ citrate-phosphate buffer. The available diffusion area between compartments was $0.785 \mathrm{~cm}^{2}$. The stirring rate and temperature of the receptor were respectively maintained at $600 \mathrm{rpm}$ and $37^{\circ} \mathrm{C}$. At appropriate intervals, $300-\mu \mathrm{L}$ aliquots of the receptor medium were withdrawn and immediately replaced with equal volumes of fresh buffer. The cumulative amounts of ALA and mALA were determined by HPLC.

\section{In vivo skin permeation examined through confocal laser scanning microscopy (CLSM)}

Localization of ALA and mALA within nude mouse skin was determined by CLSM after in vivo topical administration. A glass cylinder with an available area of $0.785 \mathrm{~cm}^{2}$ was attached to the back skin of a nude mouse with glue. ALA or mALA in the vehicles at a concentration of $38 \mathrm{mM}$ was added to the cylinder. The application times of the vehicle were 2, 4, and 8 hours. After excising the skin onto which the vehicle was applied, the skin surface was washed 10 times with a cotton cloth immersed in methanol. The skin samples obtained were examined for PpIX fluorescence images by CLSM. The skin thickness was optically scanned at about $10-\mu \mathrm{m}$ increments through the $Z$-axis of a Leica TCS SP2 confocal microscope (Wetzlar, Germany). Optical excitation was carried out with a 488-nm argon laser beam, and the fluorescence emission was detected at $590 \mathrm{~nm}$.

\section{In vivo skin irritation test}

A $0.6-\mathrm{mL}$ aliquot of the nanoemulsions was uniformly spread over a sheet of non-woven polyethylene cloth $(1.5 \times 1.5 \mathrm{~cm})$, which was then applied to the back area of a nude mouse. The polyethylene cloth was fixed with Tegaderm ${ }^{\circledR}$ adhesive dressing (3M, USA) and Fixomull ${ }^{\circledR}$ stretch adhesive tape (Beiersdorf AG, Germany). After 24 hours, the cloth was removed, and the treated skin area was swabbed clean with a cotton wool swab. Thirty minutes after withdrawing the vehicle, TEWL, colorimetric parameters, and the $\mathrm{pH}$ of the applied skin were measured. TEWL was recorded using a Tewameter ${ }^{\circledR}$ (TM300, Courage and Khazaka, Köln, Germany). Measurements taken at a stable level were performed $30 \mathrm{~s}$ after application of the TEWL probe to the skin. TEWL was automatically calculated and expressed in $\mathrm{g} / \mathrm{m}^{2} / \mathrm{h}$. A spectrocolorimeter (CD100, Yokogawa Electrical, Tokyo, Japan) was used to measure the skin erythema (a*). The skin surface $\mathrm{pH}$ was determined using a Skin-pH-Meter ${ }^{\circledR}$ PH 905 (Courage and Khazaka, Germany). An adjacent untreated site was used as a baseline standard for each determination. The temperature and relative humidity in the laboratory were respectively maintained at $26^{\circ} \mathrm{C}$ and $55 \%$.

\section{Statistical analysis}

A statistical analysis of differences between different treatments was performed using unpaired $t$ test. A 0.05 level of probability was taken as the level of significance. An analysis of variance (ANOVA) test was also used if necessary. 


\section{Results \\ Determination of the size and zeta potential}

Both $\mathrm{O} / \mathrm{W}$ and $\mathrm{W} / \mathrm{O}$ emulsions were prepared as nanocarriers for ALA and its prodrug. The average diameter and zeta potential of the resulting products without drugs were first determined and are presented in Table 1. The droplet size of $\mathrm{O} / \mathrm{W}$ emulsions ranged $216 \sim 256 \mathrm{~nm}$. The size analysis showed a mean diameter of $256 \mathrm{~nm}$ for soybean oil-loaded emulsions (W1). Replacement of the soybean oil by squalene (W2) led to a reduction in the size from 256 to $238 \mathrm{~nm}(P<0.05)$. The addition of $\alpha$-terpineol $(4 \%)$ to the soybean oil emulsions produced systems (W3) with a smaller size of $216 \mathrm{~nm}(P<0.05)$. Contrary to the results of the $\mathrm{O} / \mathrm{W}$ emulsions, the size of the squalene systems $(\mathrm{O} 2)$ was significantly larger $(P<0.05)$ than that of soybean oil-prepared ones $(\mathrm{O} 1)$ in $\mathrm{W} / \mathrm{O}$ emulsions. As depicted in Table 1, zeta potentials of most systems were negative. O/W emulsions with soybean oil (W1) exhibited a surface charge of $-20 \mathrm{mV}$, which was significantly lower $(P<0.05)$ than that of emulsions containing squalene $(-25 \mathrm{mV}, \mathrm{W} 2)$. The addition of $\alpha$-terpineol (W3) did not affect the zeta potential $(P>0.05)$. The absolute zeta potential of $\mathrm{W} / \mathrm{O}$ emulsions was approximately zero, with restricted negative charges at the interface of the soybean oil and squalene systems (O1 and $\mathrm{O} 2)$.

After loading ALA within the developed O/W systems, the mean droplet size did not significantly increase $(P>0.05)$ as shown in Table 2. However, with mALA, the size significantly increased $(P<0.05)$ after it was loaded into $\mathrm{O} / \mathrm{W}$ emulsions. The incorporation of ALA or mALA into drug-free $\mathrm{W} / \mathrm{O}$ emulsions resulted in a significant increase in the average size $(P<0.05)$. The loading of ALA showed a more-prominent increase in size compared to mALA in W/O carriers.

Table I The characterization of the oil-in-water $(\mathrm{O} / \mathrm{W})$ and waterin-oil (W/O) nanoemulsions by droplet size and zeta potential

\begin{tabular}{llrl}
\hline Code & Type & Size $(\mathrm{nm})$ & \multicolumn{1}{l}{$\begin{array}{l}\text { Zeta potential } \\
(\mathrm{mV})\end{array}$} \\
\hline WI & O/W with soybean oil & $256.3 \pm 5.5$ & $-19.9 \pm 0.8$ \\
W2 & O/W with squalene & $238.7 \pm 8.1$ & $-25.2 \pm 0.5$ \\
W3 & O/W with soybean oil & $216.2 \pm 3.7$ & $-19.0 \pm 0.6$ \\
& and terpineol & & \\
O1 & W/O with soybean oil & $33.6 \pm 1.6$ & $-2.0 \pm 0.4$ \\
O2 & W/O with squalene & $125.0 \pm 2.7$ & $-6.0 \pm 0.2$ \\
O3 & W/O with soybean oil & $18.1 \pm 0.3$ & $0.4 \pm 0.2$ \\
& and terpineol & & \\
\hline
\end{tabular}

Note: Each value represents the mean $\pm S D(n=3)$.

\section{Encapsulation efficiency of drugs in the emulsions}

The entrapment of ALA and mALA in the inner phase of $\mathrm{O} / \mathrm{W}$ emulsions was examined, and results are given in Table 3. Drug encapsulation in W/O systems was not detected, since no phase separation was observed after ultracentrifugation. Nevertheless, it is believed that most of the ALA and mALA molecules resided in the inner phase of W/O systems because of their high solubility in water. As shown in Table 3, a greater amount of mALA was incorporated in oil droplets compared with ALA $(P<0.05)$. More than half of the loaded amount of mALA was entrapped in the oil phase. Drugs could partition into soybean oil more easily than into squalene $(P<0.05)$. The incorporation of $\alpha$-terpineol resulted in a slight but significant decrease $(P<0.05)$ in drug encapsulation.

\section{Molecular environment of the emulsions}

The absorption bands of Nile red varied in shape, position, and intensity with the nature of the environment. The emission maximum of Nile red was near $600 \mathrm{~nm}$. The emission spectra of Nile red in $\mathrm{O} / \mathrm{W}$ emulsions are shown in Figure 1. The fluorescence is quenched in aqueous media and more-hydrophilic environments. ${ }^{16}$ The results indicated an increasing trend of hydrophilicity of $\mathrm{W} 2>\mathrm{W} 1>\mathrm{W} 3$. Squalene created a more-hydrophilic inner phase for emulsions compared to soybean oil. $\alpha$-Terpineol incorporation enhanced the lipophilicity of the systems.

\section{In vitro skin permeation}

The skin permeation of ALA and mALA was evaluated using Franz diffusion cells. Figure 2 shows the permeation kinetics of ALA (Figure 2A) and mALA (Figure 2B) from $\mathrm{O} / \mathrm{W}$ dispersions. The control vehicle for both drugs was DDW. The cumulative amounts of drugs at different times in the receptor are shown, and the fluxes $\left(\mathrm{nmol} / \mathrm{cm}^{2} / \mathrm{h}\right)$ calculated from the slopes are summarized in Table 4. The drug that permeates across the skin in an in vitro status dictates the amount available for subcutaneous or deeper skin tissues and systematic circulation. For the control group, the fluxes of ALA and mALA were 70 and $43 \mathrm{nmol} / \mathrm{cm}^{2} / \mathrm{h}$, respectively. Most of the cumulative amount-time profiles shown in Figure 2 followed a zero-order equation, except the W1 formulations with ALA, for which the cumulative amount gradually leveled off after 12 hours of application. The in vitro studies showed that soybean oil-containing $\mathrm{O} / \mathrm{W}$ emulsions (W1 and W3) enhanced ALA penetration by 2.6 -fold compared with the corresponding control. 
Table 2 The droplet size of the oil-in-water (O/W) and water-in-oil (W/O) nanoemulsions in the presence of 5-aminolevulinic acid (ALA) or methyl (m)ALA

\begin{tabular}{llccc}
\hline Code & Type & Without drug & ALA & mALA \\
\hline WI & O/W with soybean oil & $256.3 \pm 5.5$ & $258.6 \pm 2.3$ & $269.3 \pm 5.8$ \\
W2 & O/W with squalene & $238.7 \pm 8.1$ & $237.6 \pm 5.6$ & $253.9 \pm 5.7$ \\
W3 & O/W with soybean oil and terpineol & $216.2 \pm 3.7$ & $218.5 \pm 1.3$ & $231.9 \pm 1.2$ \\
O1 & W/O with soybean oil & $33.6 \pm 1.6$ & $57.7 \pm 2.0$ & $41.1 \pm 0.5$ \\
O2 & W/O with squalene & $125.0 \pm 2.7$ & $424.6 \pm 33.5$ & $282.1 \pm 15.9$ \\
O3 & W/O with soybean oil and terpineol & $18.1 \pm 0.3$ & $64.7 \pm 1.6$ & $26.1 \pm 3.3$ \\
\hline
\end{tabular}

Note: Each value represents the mean \pm SD $(n=3)$.

Another observation was the significant inter-subject variation of flux values of the ALA control group. This variation was reduced after ALA incorporation into the nanoemulsions. Squalene-containing O/W emulsions exhibited a low ALA flux of $10 \mathrm{nmol} / \mathrm{cm}^{2} / \mathrm{h}$. Moreover, all O/W systems showed lower mALA permeation $(P<0.05)$ compared with the control vehicle. The in vitro permeation of ALA and mALA from W/O emulsions is depicted in Figure 3A and $3 \mathrm{~B}$, respectively. Flux values are given in Table 4 . The $\mathrm{W} / \mathrm{O}$ nanocarriers turned out to be less potent in delivering drugs compared to the $\mathrm{O} / \mathrm{W}$ systems. The drug accumulation from $\mathrm{W} / \mathrm{O}$ emulsions was lower than that from the control solution at all time points, with the squalene-containing formulations (O2) showing the least permeation.

To clarify the mechanism of skin permeation, the release of ALA and mALA across cellulose membranes, through which the drug can freely traverse, was studied, and the results are shown in Table 5. The release of ALA and mALA from emulsions exhibited slower release compared to that of the aqueous solution. Release was found to be a function of the drug molecules, with a slower release rate for mALA. There was no significant difference $(P>0.05)$ among the release rates from $\mathrm{O} / \mathrm{W}$ nanocarriers for either ALA or mALA. Preparations of drugs in W/O emulsions had much slower release $(P<0.05)$ profiles than those prepared in $\mathrm{O} / \mathrm{W}$ emulsions. The rate of total ALA released from W/O systems varied from 7 to $646 \mathrm{nmol} / \mathrm{cm}^{2} / \mathrm{h}$. The range of mALA release was from 43 to $304 \mathrm{nmol} / \mathrm{cm}^{2} / \mathrm{h}$. $\alpha$-Terpineol-containing W/O dispersions (O3) showed the highest drug release, followed

Table 3 The encapsulation efficiency (\%) of 5-aminolevulinic acid (ALA) and methyl (m)ALA in oil-in-water (O/W) nanoemulsions

\begin{tabular}{llrl}
\hline Code & Type & \multicolumn{1}{l}{ ALA } & \multicolumn{1}{l}{ mALA } \\
\hline WI & O/W with soybean oil & $16.18 \pm 1.53$ & $67.95 \pm 1.82$ \\
W2 & O/W with squalene & $6.66 \pm 5.48$ & $51.87 \pm 3.25$ \\
W3 & O/W with soybean oil & $11.44 \pm 1.22$ & $60.58 \pm 6.50$ \\
& and terpineol & & \\
\hline
\end{tabular}

Note: Each value represents the mean \pm SD $(n=3)$. by preparations with soybean oil as the external phase (O1) and those with squalene $(\mathrm{O} 2)$.

\section{In vivo skin permeation examined through CLSM}

Since W1 showed an ability to enhance ALA skin permeation, this formulation was selected as a model emulsion for further in vivo studies. Figure 4 depicts representative examples of CLSM images of nude mouse skin following in vivo topical application of ALA and mALA for 2, 4, and 8 hours. ALA is a naturally occurring amino acid that is ultimately converted into PpIX within the skin. The red fluorescence shown in the images was mainly derived from PpIX within full-thickness skin. A time-dependent increase in the fluorescence intensity was measured for all test formulations. There was no significant difference in fluorescence intensities between the ALA control solution and $\mathrm{O} / \mathrm{W}$ emulsions at each time point (Figure 4A vs B). The main difference between the two vehicles was the greater numbers of filaments, which were considered to be hair follicles, in the images of W1 application for 8 hours. The same phenomenon was observed with mALA application (Figure $4 \mathrm{C}$ vs D). For the images of mALA, the intensity of the red fluorescence was much greater from W1 than from the control at 2 hours.

The CLSM fluorescence of the accumulated PpIX observed after ALA or mALA application can give an indication of the spatial distribution. The skin thickness was further scanned at about $10-\mu \mathrm{m}$ increments for 16 fragments

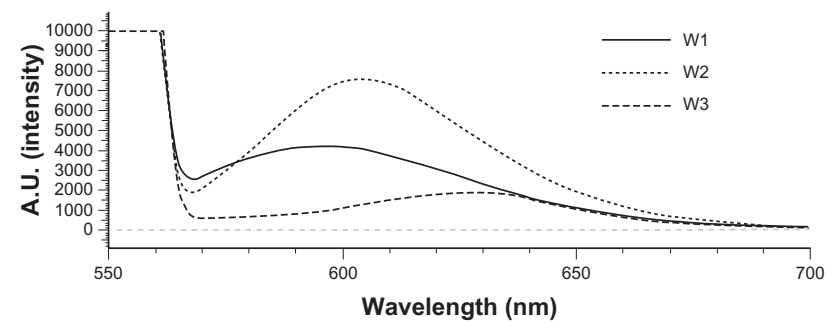

Figure I Fluorescence emission spectra of Nile red (I ppm) in oil-in-water (O/W) emulsions (WI to W3). 

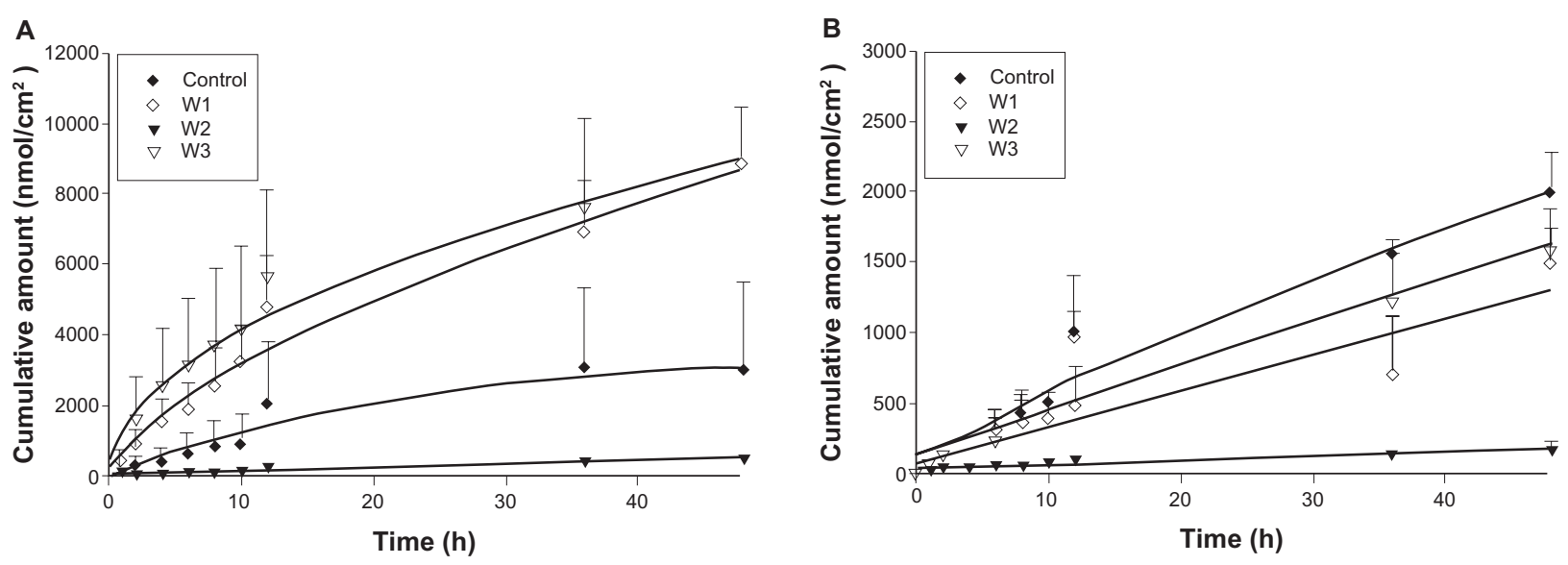

Figure 2 In vitro cumulative amount (nmol/ $\mathrm{cm}^{2}$ )-time profiles of 5-aminolevulinic acid (ALA) (A) and methyl ( $\left.\mathrm{m}\right)$ ALA (B) from the aqueous control (double-distilled water) and oil-in-water (O/W) emulsion systems (WI to W3) across porcine skin. Each value represents the mean \pm SD $(n=4)$.

starting at the surface of the skin (left to right, top to bottom). Figure 5 shows the separate images of the skin treated with test vehicles for 8 hours. Since the thicknesses of nude mouse stratum corneum (SC) and epidermis are $\sim 11$ and $\sim 18 \mu \mathrm{m}$, respectively, ${ }^{15}$ the first four fragments can be characterized as the SC and epidermal layers. The red fluorescence was generally higher in the dermis than in the SC and epidermis for all formulations tested. The intensity of the fluorescence gradually faded away with an increase in the skin depth. The extent and distribution of PpIX derived from ALA in the emulsions did not exceed those derived from the control vehicle (Figure 5A vs B). Representative fluorescence staining indicated enhanced mALA penetration into deeper skin layers after application of the nanocarrier systems compared to the control (Figure $5 \mathrm{C}$ vs D). According to the sole image with red fluorescence in Figure 5C and 5D, the penetration depth of PpIX from mALA could be increased from $100 \mu \mathrm{m}$ (control) to $140 \mu \mathrm{m}$ (W1), which would reach the layer of lower dermis. The administration of mALA O/W emulsions extensively and homogeneously increased the fluorescence intensity of the skin. It can be seen that the fluorescence levels

Table 4 The in vitro flux $\left(\mathrm{nmol} / \mathrm{cm}^{2} / \mathrm{h}\right)$ of 5 -aminolevulinic acid $(A L A)$ and methyl (m)ALA from oil-in-water (O/W) and waterin-oil (W/O) nanoemulsions via porcine skin

\begin{tabular}{lcr}
\hline Code & \multicolumn{1}{c}{ ALA } & \multicolumn{1}{c}{ mALA } \\
\hline Control & $69.75 \pm 55.87$ & $42.57 \pm 5.80$ \\
WI & $180.24 \pm 29.89$ & $25.85 \pm 8.53$ \\
W2 & $10.23 \pm 0.83$ & $2.83 \pm 1.03$ \\
W3 & $176.14 \pm 47.32$ & $33.02 \pm 5.09$ \\
OI & $11.03 \pm 3.92$ & $5.72 \pm 0.20$ \\
O2 & $1.36 \pm 0.15$ & $4.15 \pm 2.65$ \\
O3 & $34.63 \pm 8.82$ & $7.91 \pm 2.47$ \\
\hline
\end{tabular}

Note: Each value represents the mean $\pm S D(n=4)$. at the depths of the first six fragments for mALA emulsions mainly came from hair follicles, indicating the importance of shunt routes.

\section{In vivo skin irritation test}

To ensure that the topical preparations are innocuous, it is necessary to examine possible irritation caused by the emulsions. TEWL, erythema, and $\mathrm{pH}$ were used to evaluate the preliminary safety of $\mathrm{W} 1$ and $\mathrm{W} 3$ in vivo. The $\Delta$ value (the value of the treated site minus the value of an adjacent site) was determined after 24 hours of application, as shown in Figure 6. No significant skin irritation was detected when the bar of the standard deviation (SD) passes across the zero line in Figure 6. We did not see an irritant response with these formulations, suggesting tolerance of the skin to the topically applied vehicles. The incorporation of $\alpha$-terpineol (W3) induced no skin change $(P>0.05)$. Close inspection of the underlying application site also demonstrated that the emulsions did not induce visible skin reddening or disruption.

\section{Discussion}

The present work attempted to develop nanoemulsions to enhance the permeation of ALA and mALA via the skin. The effects of different oil compositions on drug delivery were compared. It was found that emulsions with different matrices provided various functions for modulating drug permeation, with soybean oil-loaded $\mathrm{O} / \mathrm{W}$ emulsions exhibiting the greatest enhancement. The influences of these nanocarriers on the delivery of ALA and its prodrug were also distinct from each other.

The principal components of soybean oil are glycerides with polyunsaturated fatty acids including oleic, linoleic, 
A

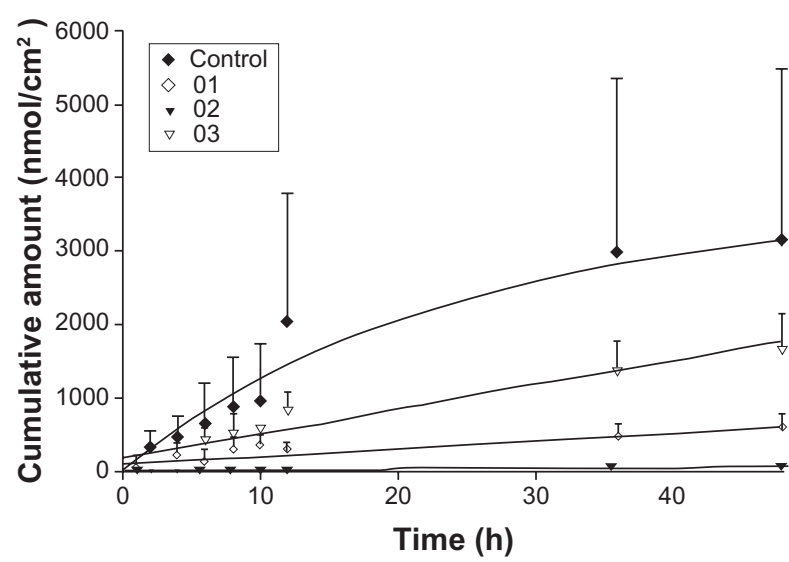

B

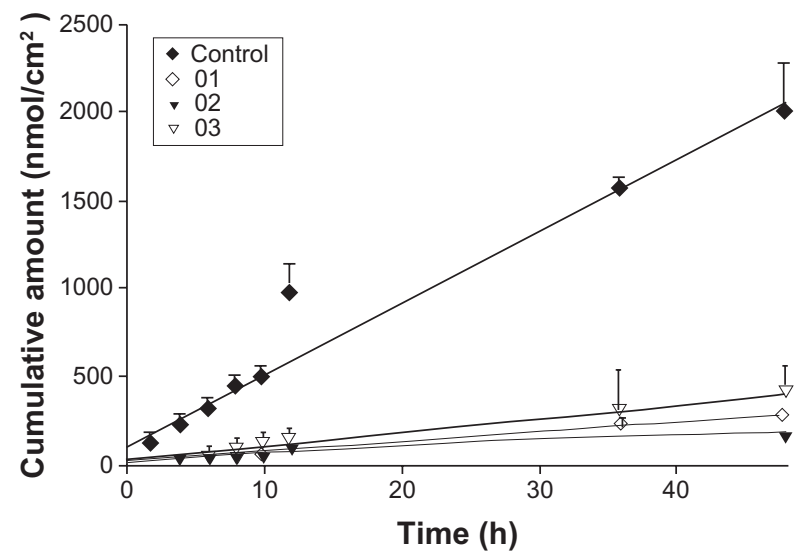

Figure 3 In vitro cumulative amount ( $\mathrm{nmol} / \mathrm{cm}^{2}$ )-time profiles of 5 -aminolevulinic acid (ALA) (A) and methyl (m)ALA (B) from the aqueous control (double-distilled water) and water-in-oil (W/O) emulsion systems $(O /$ to $O 3)$ across porcine skin. Each value represents the mean \pm SD $(n=4)$.

and linolenic acids. ${ }^{17}$ Squalene is an all-trans isoprenoid containing 6 isoprene units, and is a naturally occurring substance found in humans. There was no pronounced difference in the droplet size between the $\mathrm{O} / \mathrm{W}$ emulsions composed of soybean oil (W1) and squalene (W2), although soybean oil produced larger droplets. A significant difference was observed in the W/O emulsions, with squalene (O2) showing 4-fold larger droplets compared to soybean oil (O1). This may indicate that the emulsifier system with a determined hydrophile-lipophile balance (HLB) used in W/O emulsions (Span 80 and Brij 98) could completely emulsify and stabilize the aqueous phase and soybean oil but not squalene. The addition of the permeation enhancer, $\alpha$-terpineol, reduced the droplet size. This is because the stabilization and mixing of the two phases with these emulsifiers were easier in the $\alpha$-terpineol-containing system, ${ }^{18}$ resulting in the production of smaller droplet sizes.

The negative surface charge shown by $\mathrm{O} / \mathrm{W}$ emulsions is believed to have resulted in the ionization of Myverol. Some free fatty acids derived from the hydrolysis of

Table 5 The in vitro release rate $\left(\mathrm{nmol} / \mathrm{cm}^{2} / \mathrm{h}\right)$ of 5 -aminolevulinic acid (ALA) and methyl (m)ALA from oil-in-water (O/W) and water-in-oil (W/O) nanoemulsions via porcine skin

\begin{tabular}{lll}
\hline Code & ALA & mALA \\
\hline Control & $1850.76 \pm 40.95$ & $390.30 \pm 32.69$ \\
WI & $1642.24 \pm 195.00$ & $223.55 \pm 4.04$ \\
W2 & $1411.02 \pm 41.45$ & $226.92 \pm 25.47$ \\
W3 & $1410.61 \pm 62.34$ & $207.83 \pm 27.27$ \\
OI & $16.55 \pm 1.62$ & $101.55 \pm 18.38$ \\
O2 & $6.68 \pm 1.13$ & $43.02 \pm 4.14$ \\
O3 & $645.79 \pm 31.10$ & $303.74 \pm 70.61$ \\
\hline
\end{tabular}

Note: Each value represents the mean $\pm S D(n=4)$. monoglycerides in Myverol may have occurred, contributing to the negative charge at the interface. The zeta potential of squalene-containing formulations (W2) indicated that additional negative charges existed compared to soybean oil-containing ones (W1). This means that more Myverol may have been exposed at the interface. Myverol is characterized as a lipophilic emulsifier. According to the results of the analysis of the molecular environment, the lipophilicity of the oil droplets was less with squalene than with soybean oil. Myverol molecules tended to escape the inner squalene core with its low lipophilicity, thus exposing themselves at the interface. Another reason is the similarity of the composition between Myverol and soybean oil (glycerides), leading to the inclusion of Myverol in the inner soybean oil phase. The zeta potential of the W/O systems was nearly zero since Span 80 and Brij 98 are both non-ionic surfactants.

The $\log P$ (octanol/water partition coefficient) of ALA is negative (-1.5), as is that of the methyl ester $(-0.9) \cdot{ }^{19}$ Hence both ALA and mALA are basically hydrophilic. Because of the lipophilic matrix of $\mathrm{O} / \mathrm{W}$ emulsions, hydrophilic agents are expected to be poorly entrapped within the inner phase. It is surprising that the $\mathrm{O} / \mathrm{W}$ nanocarriers could entrap both drugs to a certain extent, especially mALA. As ALA is an amphoteric molecule, it probably strongly interacts with the emulsifier layers of the emulsions. ${ }^{20}$ This effect may have been more significant for mALA molecules. Another possibility is that some hydrophilic substances such as CAT-1 and penciclovir can be easily localized within the PF68 layer of the nanoparticles. ${ }^{21,22}$ mALA may have largely been embedded in the interface of the $\mathrm{O} / \mathrm{W}$ emulsions, resulting in a certain increase in the mean droplet size over the drug-free dispersions. This phenomenon was not detected for ALA with 

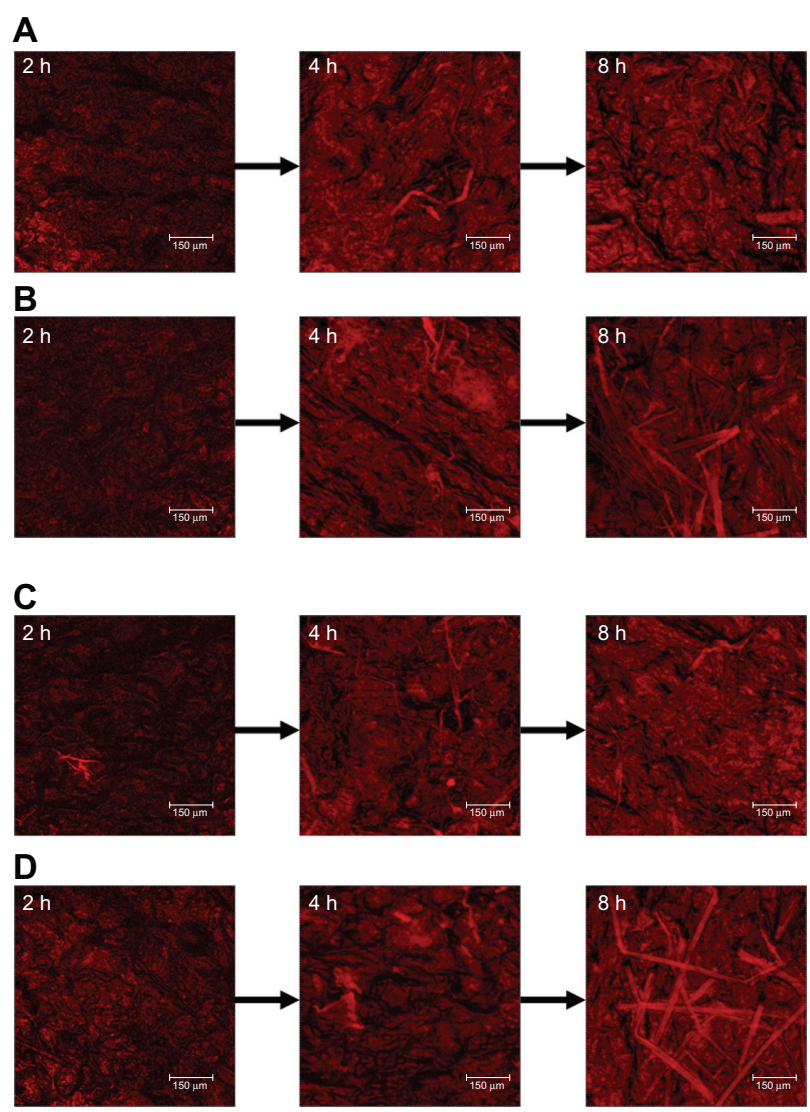

Figure 4 Confocal laser scanning microscopic (CLSM) micrographs of nude mouse skin after the in vivo topical administration of 5-aminolevulinic acid (ALA) for 2, 4, and 8 hours (left image to right image) from the aqueous control (double-distilled water) (A), ALA from oil-in-water (O/W) soybean oil emulsions (WI) (B), methyl (m)ALA from the aqueous control (double-distilled water) (C), and mALA from O/W soybean oil emulsions (WI) (D).

its lower encapsulation. Soybean oil provided greater drug encapsulation efficiency than did squalene. Soybean oil is a mixture with different fatty acids, whereas squalene is a pure compound. Mixed lipids always form less densely packed structures which should favor drug incorporation. ${ }^{23}$ In the W/O nanocarriers, ALA-loaded formulations were composed of larger droplets compared with mALA-loaded formulations. Since ALA demonstrated a higher water solubility than mALA, most of the ALA molecules may have been located in the aqueous core of the $\mathrm{W} / \mathrm{O}$ emulsions. This may have increased the dimension of the aqueous phase. On the other hand, mALA may have largely resided in the interface. The increase in size was thus confined to a certain range.

It is evident that the successful topical application of ALA for epidermal tumors would require application times of at least 24-48 hours. ${ }^{24}$ The aqueous control of ALA may have failed to achieve this duration because of the restricted increase in the in vitro cumulative amount after a 12-hour application. This disadvantage was improved by using the
A

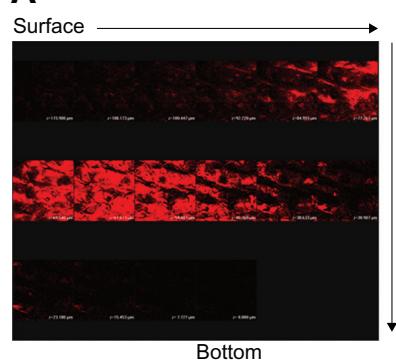

Bottom

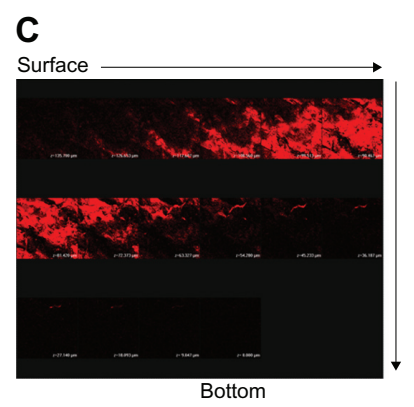

B

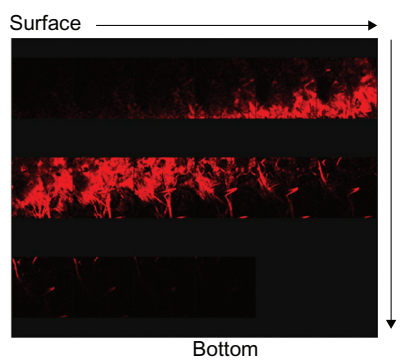

D

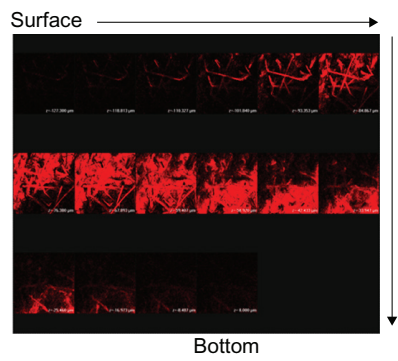

Figure 5 Confocal laser scanning microscopic (CLSM) micrographs of nude mouse skin after the in vivo topical administration of 5-aminolevulinic acid (ALA) via the skin for 8 hours from the aqueous control (double-distilled water) (A), ALA from oil-in-water (O/W) soybean oil emulsions (WI) (B), methyl (m)ALA from the aqueous control (double-distilled water) (C), and mALA from O/W soybean oil emulsions (WI) (D). The skin specimen was viewed by CLSM at $\sim 10-\mu \mathrm{m}$ increments by a separate $X, Y$-sections at each $Z$-axis from the skin surface to the bottom as the arrows indicate (left to right, top to bottom).

$\mathrm{O} / \mathrm{W}$ emulsions, especially W1. Another drawback for topical ALA delivery is the marked inter- and intra-subject variations that occur in human skin. ${ }^{25} \mathrm{~A}$ similar result was observed in the present work for the ALA-loaded aqueous control. With no reproducible control over ALA permeation to neoplastic lesions, it is inevitable that potential variations in therapeutic outcomes after PDT may become difficult to reconcile. ${ }^{7}$ The nanoemulsions attenuated the high variation that occurred with the ALA aqueous solution. ALA ester derivatives would be expected to cross cellular membranes more easily than ALA. That was not the case in this study, since mALA demonstrated lower in vitro permeation across the skin compared with ALA with the $\mathrm{O} / \mathrm{W}$ emulsions. This can be attributed to the fact that ALA was mainly dissolved in the aqueous external phase and, hence, was immediately available for release. The mALA molecules were dispersed in the interface of the droplets and, therefore, had to partition into the external phase before diffusing to the skin surface for release. ${ }^{26}$ The encapsulation efficiency and drug release profiles confirm this speculation. It seems that mALA could overcome the concentration gradient threshold before a significant permeation across the skin could be induced.

A previous study ${ }^{27}$ indicated that liposomes with lipid compositions similar to the SC increase ALA permeation. 


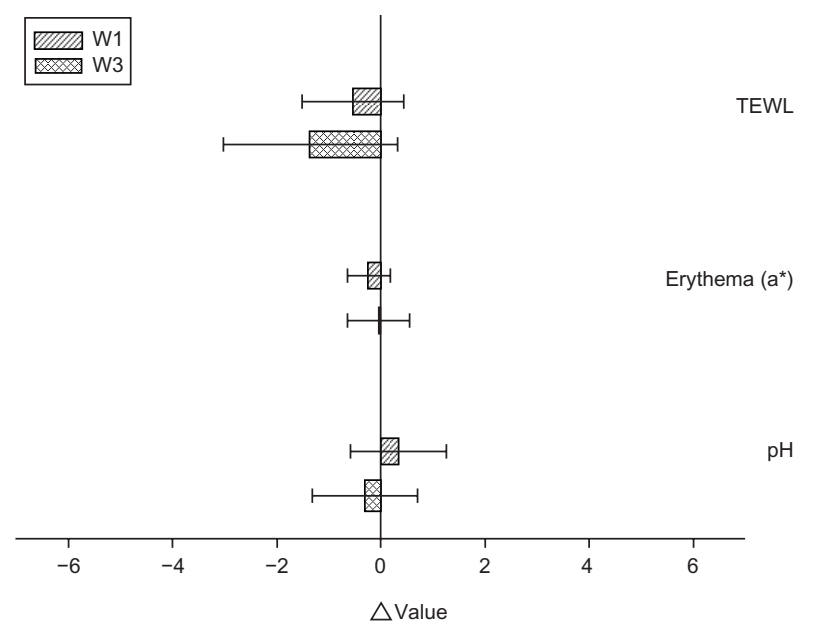

Figure 6 In vivo skin irritation examination determined by transepidermal water loss (TEWL), $\mathrm{pH}$ value, and erythema $\left(\mathrm{a}^{*}\right)$ after a 24-hour application of topically applied oil-in-water (O/W) soybean oil emulsion systems (WI and W3).

Notes: The $\Delta$ value indicates the value of the treated site minus the value of an adjacent untreated site. All data are presented as the mean of 4 experiments \pm SD.

Squalene is a structurally unique compound that is one of the main components (about 13\%) of skin surface lipids. ${ }^{28}$ Our results showed that squalene-containing $\mathrm{O} / \mathrm{W}$ emulsions did not efficiently deliver ALA into the skin. This indicates the importance of oil components to modulate the percutaneous penetration of ALA and mALA. Penetration enhancers may alter the organization of the intercellular lipids of the SC, thereby facilitating the skin permeation of ALA. That was not the case in the present work, since $\alpha$-terpineol inclusion in O/W systems (W3) did not further increase drug permeation from soybean oil-containing emulsions (W1). This may have been due to the existence of $\alpha$-terpineol in the inner phase. Thus $\alpha$-terpineol could not directly interact with the SC lipids. Contrary to this result, a significant increase in the drug flux was observed with $\alpha$-terpineol incorporation in the external phase of $\mathrm{W} / \mathrm{O}$ emulsions.

Lipid nanoparticles with smaller diameters are advantageous for improving the permeation of particles into the skin. ${ }^{29}$ Although the $\mathrm{W} / \mathrm{O}$ emulsions developed in this work had a smaller size than the $\mathrm{O} / \mathrm{W}$ carriers, the drug flux from $\mathrm{W} / \mathrm{O}$ emulsions was relatively lower than that from $\mathrm{O} / \mathrm{W}$ ones. This indicates that there was no influence of the emulsion droplet size on the skin penetration of ALA and its ester. The release rate was determined to govern the skin permeation of ALA and mALA after comparing the fluxes of $\mathrm{O} / \mathrm{W}$ and $\mathrm{W} / \mathrm{O}$ emulsions. A high ALA release rate would promote high skin permeation and therefore be advantageous for therapy. ${ }^{30}$ It can be seen that the emulsions decreased drug release compared with the release from the control solution. ALA and mALA are hydrosoluble, and thus have higher affinities for the aqueous phase. The $\mathrm{O} / \mathrm{W}$ type formulations contained a certain proportion of lipids, and this may have hindered the release of the incorporated water-soluble drugs. ${ }^{\text {? }}$ W/O-type formulations released ALA and mALA to a lesser extent than the $\mathrm{O} / \mathrm{W}$ type because of the required process of drug partitioning from the inner phase to the external phase and then diffusion through external phase. Another reason for the low drug release of $\mathrm{W} / \mathrm{O}$ emulsions was their high viscosity. With an increase in the oil phase of emulsions, the viscosity increases. ${ }^{31}$ It is possible that the high viscosity of the colloidal systems can play a role in lowering the overall release rate and subsequent skin permeation. ${ }^{32,33}$ There was a linear correlation between the release rate and flux of drugs from the three W/O formulations. Moreover, a decrease in the $\mathrm{W} / \mathrm{O}$ droplet size led to an increase in drug permeation. The rate of release generally increases with smaller droplets, since a small-droplet system has a larger total surface area where drug diffusion can occur. ${ }^{23,34}$

The CLSM profiles suggested that the accumulation and distribution of PpIX in skin exposed to mALA were improved by the presence of certain $\mathrm{O} / \mathrm{W}$ emulsions. Increasing the application time from 2 to 4 and 8 hours resulted in significant increases in tissue concentrations of both drugs. This is reasonable since the typical residence time of topically applied ALA is in the range of 4-8 hours. ${ }^{7}$ The earliest PpIX accumulation is in the epidermis, followed by the eccrine and apocrine glands, then hair follicles and sebaceous glands. ${ }^{35}$ There was a trend for hair follicles to express strong fluorescence in emulsion-treated skin after 8 hours of application. Previous studies ${ }^{6,36,37}$ indicated that the induction of PpIX accumulation by ALA and mALA is pronounced in hair follicles and sebaceous glands. Follicles and sebaceous glands are localized relatively deeply in the skin. ${ }^{38}$ Christiansen et $\mathrm{al}^{2}$ suggested that liposome-loaded ALA is preferentially absorbed in hair follicles. The nanoemulsions developed in this work likely exerted similar effects as liposomes.

Some in vivo studies ${ }^{39,40}$ suggested that topical application of ALA esters can result in greater PpIX accumulation than ALA. Although mALA exhibited a lower in vitro cumulative amount in the receptor compared to ALA, the in vivo CLSM results showed comparable fluorescence intensities between ALA and mALA. Moreover, no significant difference between the fluorescence intensities of ALA emulsions and the aqueous control was observed. This may have been due to the enzyme-limited metabolic process of ALA in skin. ${ }^{41,42}$ The cutaneous PpIX accumulation becomes saturated when a great amount of ALA is absorbed by the skin. The ester 
prodrug even provided a deeper PpIX distribution when delivered by the O/W soybean oil emulsions. For optimal efficacy, PDT drugs should ideally penetrate the skin to reach the target tissue at sufficiently high concentrations. Cutaneous delivery of ALA failed to produce measurable quantities within the skin, in particular for deeper dermal and subepidermal tumors. ${ }^{7,24}$ Nodular BCC is an example of cells located deeper than the epidermis. The $\mathrm{O} / \mathrm{W}$ emulsions containing soybean oil clearly promoted $m$ ALA penetration to the deeper skin compared with the aqueous solution. As a consequence, topical administration of mALA emulsions is promising for targeting lesions underlying the epidermis. Of course this does not mean that ALA in soybean oil emulsions is of no use to efficiently target skin lesions. Lopez et a ${ }^{19}$ suggested that in vitro measurement of the ALA flux into the receptor provides some evidence of its local cutaneous availability, especially in deeper tissues.

The in vitro and in vivo permeation results suggest that enhanced drug skin delivery was achieved by the $\mathrm{O} / \mathrm{W}$ soybean oil emulsions. Since the release rate from the formulations cannot explain this high skin absorption, other mechanisms may predominate the delivery of nanocarriers. These include the role of hair follicles/sebaceous glands, the enhancer effect, and an increase in thermodynamic activity. One of the features that make lipid nanocarriers interesting for dermatological applications is their tendency to preferentially penetrate and accumulate in hair follicles. ${ }^{43,44}$ Oil droplets are compatible with the sebum and are preferably transported into follicles/sebaceous glands. The higher flexibility of nanocarriers may be an advantage within the narrow slot between the hair root sheath and shaft. ${ }^{45}$ Penetration of soybean oil emulsions had a more flexible character because of the mixture of different fatty acids. Penetration of squalene droplets may have been retarded because of their rigid structure based on the ordered arrangement of the inner-phase construction. The importance of hair follicles for emulsion penetration was verified by the in vivo CLSM results for mALA. However, this effect of permeation enhancement for mALA was not detected in the in vitro receptor compartment. This was because after skin excision, the follicular volume was reduced by the contraction of the elastic fibers so that the follicles were less receptive to the applied substances. ${ }^{46,47}$

The rate-limiting step for ALA and mALA uptake into skin lies at the level of the $\mathrm{SC} .{ }^{48}$ Crossing the $\mathrm{SC}$ is essential for ALA conversion to PpIX in the skin. The nanoemulsions interacted with the lipid structure of the $\mathrm{SC}$ in such a way that facilitated the permeation of drugs across the skin. Fatty acids and glycerides can be used as effective penetration enhancers for a variety of drugs. ${ }^{49}$ Fatty acids can enter the bilayers, and perturb them by creating separate domains. The unsaturated cis configuration perturbs the lipid packing more than does a trans configuration. ${ }^{50}$ Monoolein is a mixture of glycerides and other fatty acids, mainly glycerol monooleate. It is an effective permeation enhancer for ALA. ${ }^{8,19}$ Some unsaturated fatty acids and glycerides are derived from the hydrolysis of soybean oil, meaning that this oil has a penetration enhancer role. This effect was not observed for squalene. Although intact lipid particles basically do not penetrate the SC, uptake of their components is to be expected.$^{51}$ Liquid-state droplets perturbing the lipid organization in the deeper horny layer are more effective than rigid-state vesicles in increasing skin permeation. ${ }^{23}$

For nanocarriers, issues such as the thermodynamic activity of the permeant relative to the vehicle are very important, as they determine the push into the skin. ${ }^{13}$ Differences in the internal structure of the emulsions can modify the thermodynamic activity of ALA, which could favor its partitioning into the SC. O/W soybean oil emulsions may provide higher thermodynamic activity for ALA and mALA than squalene-containing carriers. A detailed investigation of the enhancing mechanisms of drug permeation was not the main effort in this study. Although the follicular pathway and enhancer effect succeeded in elucidating the penetration of ALA and mALA from the nanocarriers, the skin targeting mechanisms are unclear, and further investigations are needed in the future.

In topical formulations, the irritation issue is not considered in many cases. It is important to find a balance between skin permeation and skin irritation of a particular vehicle for practical use. TEWL was used to assess the degree of SC disruption. The $\mathrm{a}^{*}$-coordinate of colorimetry (which indicates erythema) was demonstrated to correlate well with inflammation of the skin. ${ }^{52}$ It was found that although W1 and W3 almost tripled the flux of ALA, no or negligible increases in TEWL and $a^{*}$ were detected. This result suggests that the barrier function of the skin was not compromised by the preparations. Contact between the skin and the formulations may provoke acceptable tolerance.

\section{Conclusion}

ALA and mALA were formulated at equimolar concentrations in $\mathrm{O} / \mathrm{W}$ and $\mathrm{W} / \mathrm{O}$ nanoemulsions for skin delivery. We showed that not only the choice of the drug, but also the emulsion type and the oil phase are all important considerations when attempting to optimize topical drug permeation. The $\mathrm{O} / \mathrm{W}$ soybean oil dispersions were most promising because of their 
ability to exert the highest in vitro ALA flux. The uniformity of drug flux by the emulsions was improved when compared to the aqueous control. The addition of $\alpha$-terpineol, a penetration enhancer, as a part of the oil phase did not further increase drug permeation via the skin. The optimized formulations could also deliver mALA to deeper layers of the skin. The release rate of the drugs from the inner phase was the main mechanism governing the skin permeation from $\mathrm{W} / \mathrm{O}$ emulsions. On the other hand, follicular routes, a penetration enhancer effect, and increases in thermodynamic activity were possible factors predominating the permeation enhancement of $\mathrm{O} / \mathrm{W}$ soybean oil emulsions. The skin irritation test indicated negligible skin disruption and acceptable safety of the prepared nanocarriers.

\section{Disclosures}

The authors declare no conflicts of interest.

\section{References}

1. Wheless L, Black J, Alberg AJ. Nonmelanoma skin cancer and the risk of second primary cancers: a systemic review. Cancer Epidemiol Biomarkers Prev. 2010;19:1686-1695.

2. Christiansen K, Bjerring P, Troilius A. 5-ALA for photodynamic photorejuvenation - Optimization of treatment regime based on normal-skin fluorescence measurements. Lasers Surg Med. 2007;39:302-310.

3. De Rosa FS, Bentley MVLB. Photodynamic therapy of skin cancers: sensitizers, clinical studies and future directives. Pharm Res. 2000; 17:1447-1455.

4. Blume JE, Oseroff AR. Aminolevulinic acid photodynamic therapy for skin cancers. Dermatol Clin. 2007;25:5-14.

5. Rodriguez L, Batlle A, Di Venosa G, et al. Mechanisms of 5-aminolevulinic acid ester uptake in mammalian cells. Br J Pharmacol. 2006;147:825-833.

6. De Bruijn HS, Meijers C, van der Ploeg-van den Heuvel A, Sterenborg HJCM, Robinson DJ. Microscopic localisation of protoporphyrin IX in normal mouse skin after topical application of 5-aminolevulinic acid or methyl 5-aminolevulinate. J Photochem Photobiol B: Biol. 2008; 92:91-97.

7. McCarron PA, Donnelly RF, Zawislak A, Woolfson AD. Design and evaluation of a water-soluble bioadhesive patch formulation for cutaneous delivery of 5-aminolevulinic acid to superficial neoplastic lesions. Eur J Pharm Sci. 2006;27:268-279.

8. Steluti R, De Rosa FS, Collett J, Tedesco AC, Bentley MVLB. Topical glycerol monooleate/propylene glycol formulations enhance 5-aminolevulinic acid in vitro skin delivery and in vivo protoporphyrin IX accumulation in hairless mouse skin. Eur J Pharm Biopharm. 2005; 60:439-444.

9. Fang YP, Tsai YH, Wu PC, Huang YB. Comparison of 5-aminolevulinic-encapsulated liposome versus ethosome for skin delivery for photodynamic therapy. Int J Pharm. 2008;356:144-152.

10. Derycke ASL, de Witte PAM. Liposomes for photodynamic therapy. Adv Drug Deliv Rev. 2004;56:17-30.

11. Casas A, Batlle A. Aminolevulinic acid derivatives and liposome delivery as strategies for improving 5-aminolevulinic acid-mediated photodynamic therapy. Curr Med Chem. 2006;13:1157-1168.

12. Solans C, Izquierdo P, Nolla J, Azemar N, García-Celma MJ. Nano-emulsions. Curr Opin Colloid Interface Sci. 2005;10:102-110.

13. Izquierdo P, Wiechers JW, Escribano E, et al. A study on the influence of emulsion droplet size on the skin penetration of tetracaine. Skin Pharmacol Physiol. 2007;20:263-270.
14. Weyenberg W, Filev P, Van den Plas D, et al. Cytotoxicity of submicron emulsions and solid lipid nanoparticles for dermal application. Int $J$ Pharm. 2007;337:291-298.

15. Shen SC, Lee WR, Fang YP, Hu CH, Fang JY. In vitro percutaneous absorption and in vivo protoporphyrin IX accumulation in skin and tumors after topical 5-aminolevulinic acid application with enhancement using an erbium:YAG laser. J Pharm Sci. 2006;95:929-938.

16. Jores K, Haberland A, Wartewig S, Mäder K, Mehnert W. Solid lipid nanoparticles (SLN) and oil-loaded SLN studied by spectrofluorometry and Raman spectroscopy. Pharm Res. 2005;22:1887-1897.

17. Wang JJ, Hung CF, Yeh CH, Fang JY. The release and analgesic activities of morphine and its ester prodrug, morphine propionate, formulated by water-in-oil nanoemulsions. J Drug Target. 2008;16:294-301.

18. Hwang TL, Fang CL, Chen CH, Fang JY. Permeation enhancercontaining water-in-oil nanoemulsions as carriers for intravesical cisplatin delivery. Pharm Res. 2009;26:2314-2323.

19. Lopez RFV, Bentley MVLB, Delgado-Charro MB, Guy R. Optimization of aminolevulinic acid delivery by iontophoresis. J Control Release. 2003;88:65-70.

20. Nielsen HM, Aemisegger C, Burmeister G, Schuchter U, Gander B. Effect of oil-in-water emulsions on 5-aminolevulinic acid uptake and metabolism to PpIX in cultured MCF-7 cells. Pharm Res. 2004;21: 2253-2260.

21. Küchler S, Abdel-Mottaleb M, Lamprecht A, Radowski MR, Haag R, Schäfer-Korting M. Influence of nanocarrier type and size on skin delivery of hydrophilic agents. Int J Pharm. 2009;377:169-172.

22. Lv Q, Yu A, Xi Y, et al. Development and evaluation of penciclovirloaded solid lipid nanoparticles for topical delivery. Int J Pharm. 2009; 372:191-198.

23. Schäfer-Korting M, Mehnert W, Korting H. Lipid nanoparticles for improved topical application of drugs for skin diseases. Adv Drug Deliv Rev. 2007;59:427-443.

24. Bretschko E, Szeimies RM, Landthaler M, Lee G. Topical 5-aminolevulinic acid for photodynamic therapy of basal cell carcinoma. Evaluation of stratum corneum permeability in vitro. J Control Release. 1996;42:203-208.

25. Gerritsen MJP, Smits T, Kleinpenning MM, van de Kerkhof PCM, van Erp PEJ. Pretreatment to enhance protoporphyrin IX accumulation in photodynamic therapy. Dermatology. 2009;218:193-202.

26. Donnelly RF, McCarron PA, Woolfson AD. Drug delivery of aminolevulinic acid from topical formulations intended for photodynamic therapy. Photochem Photobiol. 2005;81:750-767.

27. Pierre MBR, Tedesco AC, Marchetti JM, Bentley MVLB. Stratum corneum lipids liposomes for the topical delivery of 5-aminolevulinic acid in photodynamic therapy of skin cancer: preparation and in vitro permeation study. BMC Dermatol. 2001;1:5.

28. Huang ZR, Lin YK, Fang JY. Biological and pharmacological activities of squalene and related compounds: potential uses in cosmetic dermatology. Molecules. 2009;14:540-554.

29. Liu J, Hu W, Chen H, Ni Q, Xu H, Yang X. Isotretinoin-loaded solid lipid nanoparticles with skin targeting for topical delivery. Int J Pharm. 2007;328:191-195.

30. Lieb S, Szeimies R, Lee G. Self-adhesive thin films for topical delivery of 5-aminolevulinic acid. Eur J Pharm Biopharm. 2002;53:99-106.

31. Araújo LMPC, Thomazine JA, Lopez RFV. Development of microemulsions to topically deliver 5-aminolevulinic acid in photodynamic therapy. Eur J Pharm Biopharm. 2010;75:48-55.

32. Wu H, Ramachandran C, Weiner ND, Roessler BJ. Topical transport of hydrophilic compounds using water-in-oil nanoemulsions. Int J Pharm. 2001;222:63-75.

33. Teeranachaideekul V, Souto EB, Junyaprasert VB, Müller RH. Cetyl palmitate-based NLC for topical delivery of coenzyme $\mathrm{Q}_{10}$ Development, physicochemical characterization and in vitro release studies. Eur J Pharm Biopharm. 2007;67:141-148.

34. Hung CF, Fang CL, Liao MH, Fang JY. The effect of oil components on the physicochemical properties and drug delivery of emulsions: tocol emulsion versus lipid emulsion. Int J Pharm. 2007;335:193-202. 
35. Sakamoto FH, Tannous Z, Doukas AG, et al. Porphyrin distribution after topical aminolevulinic acid in a novel porcine model of sebaceous skin. Lasers Surg Med. 2009;41:154-160.

36. Kosaka S, Kawana S, Zouboulis CC, Hasan T, Ortel B. Targeting of sebocytes by aminolevulinic acid-dependent photosensitization. Photochem Photobiol. 2006;82:453-457.

37. Morrow DIJ, McCarron PA, Woolfson AD, et al. Novel patch-based systems for the localized delivery of ALA-esters. J Photochem Photobiol B: Biol. 2010;101:59-69.

38. Juzeniene A, Juzenas P, Ma LW, Iani V, Moan J. Topical application of 5-aminolevulinic acid, methyl 5-aminolevulinate and hexyl 5-aminolevulinate on normal human skin. Br J Dermatol. 2006;155: 791-799.

39. Tunstall RG, Barnett AA, Schofield J, et al. Porphyrin accumulation induced by 5 -aminolevulinic acid esters in tumor cells growing in vitro and in vivo. Br J Cancer. 2002;87:246-250.

40. Lopez RFV, Lange N, Guy R, Bentley MVLB. Photodynamic therapy of skin cancer: controlled drug delivery of 5-ALA and its esters. $A d v$ Drug Deliv Rev. 2004;56:77-94.

41. Casas A, Fukuda H, Batlle A. Tissue distribution and kinetics of endogenous porphyrins synthesized after topical application of ALA in different vehicles. Br J Cancer. 1999;81:13-18.

42. Tsai JC, Chen IH, Wong TW, Lo YL. In vitro/in vivo correlations between transdermal delivery of 5-aminolevulinic acid and cutaneous protoporphyrin IX accumulation and effect of formulation. $\mathrm{Br} J$ Dermatol. 2002;146:653-862.

43. Puglia C, Blasi P, Rizza L, et al. Lipid nanoparticles for prolonged topical delivery: an in vitro and in vivo investigation. Int J Pharm. 2008;357:295-304.
44. Knorr F, Lademann J, Patzelt A, Sterry W, Blume-Peytavi U, Vogt A. Follicular transport route - Research progress and future perspectives. Eur J Pharm Biopharm. 2009;71:173-180.

45. Jung S, Patzelt A, Otberg N, Thiede G, Sterry W, Lademann J. Strategy of topical vaccination with nanoparticles. J Biomed Opt. 2009;14: 021001.

46. Starcher B, Aycock RL, Hill CH. Multiple roles for elastic fibers in the skin. J Histochem Cytochem. 2005;53:431-443.

47. Rancan F, Papakostas D, Hadam S, et al. Investigation of polylactic acid (PLA) nanoparticles as drug delivery systems for local dermatotherapy. Pharm Res. 2009;26:2027-2036.

48. Lee WR, Shen SC, Pai MH, Yang HH, Yuan CY, Fang JY. Fractional laser as a tool to enhance the skin permeation of 5-aminolevulinic acid with minimal skin disruption: a comparison with conventional erbium:YAG laser. J Control Release. 2010;145:124-133.

49. Kogan A, Garti N. Microemulsions as transdermal drug delivery vehicles. Adv Colloid Interf Sci. 2006;123-126:369-385.

50. Augnst BJ, Rogers NJ, Shefter E. Enhancement of naloxone penetration through human skin in vitro using fatty acids, fatty alcohols, surfactants, sulfoxides and amides. Int J Pharm. 1986;33:225-234.

51. Lombardi Borgia S, Regehly M, Sivaramakrishnan R, et al. Lipid nanoparticles for skin penetration enhancement - correlation to drug localization within the particle matrix as determined by fluorescence and parelectric spectroscopy. J Control Release. 2005;110:151-163.

52. Lin YK, Huang ZR, Zhuo RZ, Fang JY. Combination of calcipotriol and methotrexate in nanostructured lipid carriers for topical delivery. Int J Nanomed. 2010;5:117-128.
International Journal of Nanomedicine

\section{Publish your work in this journal}

The International Journal of Nanomedicine is an international, peerreviewed journal focusing on the application of nanotechnology in diagnostics, therapeutics, and drug delivery systems throughout the biomedical field. This journal is indexed on PubMed Central, MedLine, CAS, SciSearch $\AA$, Current Contents ${ }^{\circledR} /$ Clinical Medicine,

\section{Dovepress}

Journal Citation Reports/Science Edition, EMBase, Scopus and the Elsevier Bibliographic databases. The manuscript management system is completely online and includes a very quick and fair peer-review system, which is all easy to use. Visit http://www.dovepress.com/ testimonials.php to read real quotes from published authors. 